\title{
AVALIAÇÃO DOS COMPROMETIMENTOS OCULARES EM HANSENIANOS VIRGENS DE TRATAMENTO *
}

\author{
Bárbara Celeste Rolim ** \\ Élida Marta Santos Silva ***
}

\begin{abstract}
RESUMO - Foram avaliados através da consulta de enfermagem em prevenção oftalmológica no Hospital Estadual Curupaiti, RJ, 108 hansenianos virgens de tratamento afim de detectar o nivel de comprometimento do aparelho visual, no início do tratamento clínico. Diversos foram os comprometimentos detectados, como diminuição da produção de lágrimas, paresia do músculo orbicular da pálpedra, diminuição da sensibilidade corneal e pterígio. Percebemos que a prevalência aumenta na dependência direta de fatores como: idade, forma clínica e tempo de doença. A partir desta situação, enfatiza-se a necessidade de avaliação sistemática e rotineira do aparelho visual pelos profissionais de saúde que atuam no programa de controle da endemia. Propomos que o enfermeiro assuma esta atividade, propiciando, assim atuação direta na assistência e abrindo novo campo de atuação para a categoria.
\end{abstract}

\begin{abstract}
It has been evaluated by a nursing exam in ophtalmologic prevention at Hospital Estadual Curupaiti, RJ, 108 leprosy patients without treatment before this illness, in order to detect the level of visual system complications at the first contact with health service. Many complications had been detected and we noticed that the prevalence increases in direct dependency of different factors such as: age, disease time and ceinicar forms. From this situation on, we try to emphasize the necessity of a routine and a systematic evaluation of the visual system by the health professionals who work at leprosy control programme.
\end{abstract}

\section{INTRODUÇÃO}

A hanseníase é uma doença crônica que atinge $o$ aparelho visual com alta frequência, ocasionando complicações que, em geral, se não tratada levarão à cegueira. Relatam COURTRIGHT, JOHNSON ${ }^{1}$, que estimativas da OMS revelam que a prevalência de severa perda da acuidade visual situa-se em torno de 6-10\% para contagem de dedos em 6 metros e em torno de $4-7 \%$ para contagem de dedos em 3 metros.

Se analisarmos a associação entre esta incapacidade e a insensibilidade de mãos e pés, perceberemos que o portador dessa doença nestas condições torna-se bastante vulnerável a traumatismos diversos. Assim, um bom programa de prevenção de incapacidade de mãos e pés só surtirá o efeito dese jado se o paciente puder

tar, através da integridade visual, situações saumatizantes. Contudo, este aspecto é pouco valorizado na avaliação de rotina do quadro evolutivo da hanseníase; "poucos pofissionais de saúde lembrariam de citar esta complicação se questionados sobre a hanseníase", como constata ROGERS ${ }^{2}$.

As lesões oculares hansênicas instalam-se insidiosamente com pouca ou nenhuma sintomatologia, razão pela qual são negligenciadas e não tratadas. Como consequência, o diagnóstico é tardio, quando já estão instalados quadros graves, como lagoftalmo, irites ou iridociclites que levarão à diminuição progressiva da visão ou mesmo, em último instância, à cegueira. A forma como estas lesões ocorrem, explica-se pela maneira como os olhos são afetados na moléstia. Conforme apresentado por BRAND, JOFRION ${ }^{3}$ temos:

“- exposição anormal dos olhos por comprometimento de 50 e 70 pares cranianos;

* Prêmio Isaura Barbosa Lima - 3\% lugar - 43ำ Congresso Brasileiro de Enfermagem - Curitiba-PR.

** Enfermeira do Instituto Estadual de Dermatologia Sanitária - Professora da Faculdade de Enfermagem da FESO - Teresópolis-RJ.

*** Enfermeiro do Instituto Estadual de Dermatologia Sanitária.

Trabalho coordenado pelo médico-infectologista Alfredo Marques Boechat. 
- infiltração dos olhos e anexos pelo bacilo de Hansen.

- inflamação dos olhos e anexos, secundariåmente, a infiltração pelo bacilo de Hansen;

- complicações secundárias devido a envolvimento das estruturas anexas (glândula lacrimal, pálpedra e conduto nasolacrimal)".

\subsection{Objetivos}

No trabalho ora apresentado objetivamos:

- detectar a prevalência dos compromentimentos oculares e dos anexos em hansenianos virgens de tratamento, que demandaram ao serviço de hansenologia do Hospital Estadual $\mathrm{Cu}$ rupaiti, afim de enfatizar a importância do acompanhamento preventivo de rotina;

- conhecer a interferência de outros fatores na prevalência dos compromissos oculares e dos anexos em hansenianos virgens de tratamento.

\section{SUJEITOS E METODOLOGIA}

O presente trabalho foi desenvolvido no período de 1988 a 1990, no setor de Enfermagem em Prevenção Oftalmológica do Hospital Estadual Curupaiti, no Rio de Janeiro.

O estudo considerou como amostra, do total de pacientes que demandou ao setor, 108 os quais não haviam recebido tratamento anterior para hanseníase e por isso denominados virgens de tratamento (VT).

O paciente é submetido ao exame ocular, durante a consulta de enfermagem por ocasião do início do tratamento multidrogaterápico *. O exame consta de: anamnese, dirigida para as queixas subjetivas, inspeção macroscópica, com auxílio de lupa e lanterna manual, de câmara anterior; avaliação da acuidade visual feita com a escala de Snellen; avaliação da sensibilidade corneal testada com fio dental; avaliação da produção de lágrimas através do teste de Schirmer.

A amostra estudada compunha-se de: 60 $(55,6 \%)$ homens, $48(55,4 \%)$ mulheres, cujas idades variam de 5 a 65 anos, com concentração maior $(70,1 \%)$ nas faixas de 15 a 55 anos. Segundo classificação de Madri, encontrou-se: 14
$(13,0 \%)$ de forma indeterminada - I, $26(24,1 \%)$ de forma tubercolóide - $\mathrm{T}$ e de forma dimorfa $D$, respectivamente e $42(38,8 \%)$ de forma virchorwiana - V. O tempo de doença relatado pelos pacientes variou de 1 a mais de 3 anos. Vale ressaltar, que este atributo, apesar de bastante utilizado, não é fidedigno, pois é obtido no momento da investigação epidemiológica, a partir da lembrança do paciente sobre a época do aparecimento dos primeiros sinais e sintomas da hanseníase.

\section{ANÁLISE E DISCUSSÃO DOS DADOS}

A discussão que se segue tem como eixo fundamental os comprometimentos oculares objetivos detectados na amostra. Esta variável foi correlacionada à: faixa etária, forma clínica e tempo de doença. Os dados foram distribuídos em frequência total e percentual e apresentados em tabelas simples (2x2). Em uma única tabela apresentaremos os sintomas subjetivos relatados pelos pacientes.

Ao analisarmos a Tabela 1, percebemos elevado percentual de pacientes, independente de forma clínica, relatando queixas subjetivas. Em oposição, o percentual de pacientes sem queixas é reduzido (25\%). Nota-se que os pacientes de forma virchowiana são os que mais frequentemente manifestam queixas, sendo acompanhado a seguir pelos de forma tuberculóide. Deve ser causa de atenção do profissional de saúde o significativo percentual de pacientes de forma indeterminada que apresenta queixas subjetivas. A queixa mais frequente, prurido, pode ser justificada, neste caso por uma diminuição da produção de lágrimas, quadro bastante incidente em hansenianos (Tab. 2). Este fato sugere uma correlação da queixa subjetiva ao quadro objetivo. Esta hipótese é reforçada por outros trabalhos segundo BRAND, JOFRION $^{3}$ que apresentam esta queixa com frequência. $O$ embaçamento e $o$ ardor podem estar também correlacionados ao quadro de diminuição da produção de lágrimas, embora patologias como diminuição da acuidade visual e infecções também possam determinar estes sintomas.

\footnotetext{
* A Multidrogaterapia é um novo esquema de tratamento padronizada pela OMS em 1984 e composto de 3 drogas: rifampicina, sulf ona, lamprem, administradas simultaneamente. Atualmente é o esquema de tratamento oficial do Brasil.
} 
Tabela 1 - Distribuição dos comprometimentos subjetivos por forma clínica - PO/HECU - 1991.

\begin{tabular}{|c|c|c|c|c|c|c|c|c|c|c|c|}
\hline \multirow[b]{2}{*}{ Comprometimento Subjetivo } & \multirow{2}{*}{ Forma Clínica } & \multicolumn{2}{|c|}{ I } & \multicolumn{2}{|c|}{$\Gamma$} & \multicolumn{2}{|c|}{$\mathrm{D}$} & \multicolumn{2}{|c|}{ V } & \multicolumn{2}{|c|}{ Total } \\
\hline & & № & $\%$ & № & $\%$ & № & $\%$ & № & $\%$ & No & $\%$ \\
\hline Prurido & & 04 & 8,7 & 15 & 32,6 & 09 & 19,6 & 18 & 39,1 & 46 & $.42,6$ \\
\hline Embaçamento & & 06 & 15,4 & 12 & 30,8 & 09 & 23,0 & 12 & 30,8 & 39 & 36,1 \\
\hline Ardor & & 05 & 14,3 & 08 & 22,8 & 05 & 14,3 & 17 & 48,6 & 35 & 32,4 \\
\hline Dor & & 05 & 16,7 & 07 & 23,3 & 05 & 16,7 & 13 & 43,3 & 30 & 27,7 \\
\hline Lacrime jamento & & 04 & 13,3 & 06 & 20,0 & 08 & 26,7 & 12 & 40,0 & 30 & 27,7 \\
\hline Sensação areia & & 05 & 20,0 & 07 & 28,0 & 04 & 16,0 & 09 & 36,0 & 25 & 24,0 \\
\hline Sem queixas & & 05 & 18,5 & 06 & 22,2 & 07 & 25,9 & 09 & 33,4 & 27 & 25,0 \\
\hline
\end{tabular}

Na Tabela 2 correlacionamos os comprometimentos objetivos às diferentes formas clínicas. Ao analisarmos a mesma, podemos separar os compromentimentos detectados em dois grupos distintos: os diretamente associados à hanseníase e aqueles para os quais a associação ainda não está bem explicitada. No primeiro grupo, os comprometimentos mais prevalentes foram:

$\downarrow$ do Shirmer (= diminuição da produção lacrimal) 44,4\%; paresia (= diminuição da força do músculo orbicular da pálpebra) $20,4 \%$ e diminuição da sensibilidade corneal, $20,4 \%$. A correlação entre estes comprometimentos e a hanseníase é evidente se considerarmos os macanismos de acometimentos do aparelho visual pelo "Micobacterium leprae". No segundo grupo, notamos como mais prevalentes: pterígio inicial (= espessamento da conjuntiva), $39,8 \%$ e diminuição da acuidade visual para longe, $22,2 \%$. A correlação destes comprometimentos com a hanseníase ainda não é clara, contudo, é dado comum a outros trabalhos a elevada incidência de pterígio em hansenianos.

Analisando a distribuição pelas formas clínicas, independente da correlação direta ou indireta, percebemos que os pacientes virchowianos apresentam maior comprometimento ocular.
Este fato pode ser justificado pela elevada carga bacilar e pela menor competência do sistema imunológico, propiciando maior agressão do aparelho visual, bem como a outras estruturas corpóreas. Neste grupo pode-se ainda juntar os portadores da forma dimorfas. Os pacientes tuberculóides, também apresentam significativa incidência de acometimentos. Estes devem ser sistematicamente acompanhados, pois a melhor resposta do sistema imunológico, pode propiciar aparecimento de quadros bruscos e, por vezes, irreversíveis. Os portadores de forma indeterminada, apesar da menor incidência, também apresentam comprometimentos oculares. $\mathrm{O}$ número de pacientes em todas as formas clínicas, sem comprometimento é reduzido, demonstrando que talvez seja precoce o envolvimento do aparelho visual na hanseníase. Este achado deve merecer atenção pelos profissionais de saúde, principalmente se atentarmos ao fato de que, os comprometimentos' mais incidentes só são através de um exame minucioso e específico. Logo deve ser falsa a impressão de que, "muitos pacientes, no início do tratamento não apresentam comprometimentos oculares", como é usual ouvir-se nos serviços de atendimento a. pacientes hansenianos.

Tabela 2 - Distribuição dos comprometimentos objetivos dos olhos e anexos por forma clínica PO/HECU - 1991.

\begin{tabular}{|c|c|c|c|c|c|c|c|c|c|c|c|}
\hline \multirow[b]{2}{*}{ Comprometimento Subjetivo } & Forma Clínica & \multicolumn{2}{|c|}{ I } & \multicolumn{2}{|c|}{$\mathbf{T}$} & \multicolumn{2}{|c|}{$\mathrm{D}$} & \multicolumn{2}{|c|}{ V } & \multicolumn{2}{|c|}{ Total } \\
\hline & & № &.$\%$ & № & $\%$ & № & $\%$ & № & $\%$ & № & $\%$ \\
\hline I Schirmer & & 05 & 10,4 & 15 & 31,2 & 11 & 23,0 & 17 & 35,4 & 48 & 44,4 \\
\hline Paresia & & 02 & 9,0 & 07 & 31,9 & 04 & 18,02 & 09 & 40,9 & 22 & 20,4 \\
\hline Sensibilidade corneal & & 01 & 4,5 & 04 & 18,2 & 05 & 22,8 & 12 & 54,5 & 22 & 20,4 \\
\hline Madarose parcial & & 0 & 0 & 01 & 5,6 & 05 & 17,7 & 12 & 66,7 & 18 & 16,7 \\
\hline$\downarrow$ Ac. visual p. longe & & 03 & 12,5 & 07 & 29,2 & 09 & 37,5 & 05 & 20,8 & 24 & 22,2 \\
\hline$\downarrow$ Ac. Visual p. perto & & 01 & 6,2 & 05 & 31,3 & 09 & 56,3 & 01 & 6,2 & 16 & 14,8 \\
\hline Triquíase & & 01 & 33,3 & 0 & 0 & 0 & 0 & 02 & 66,7 & 03 & 2,8 \\
\hline Lagoftalmo I (5mm) & & 0 & 0 & 01 & 33,3 & 0 & 0 & 02 & 66,7 & 03 & 2,8 \\
\hline Cegueira (unilateral) & & 0 & 0 & 01 & 50,0 & 01 & 50,0 & 0 & 0 & 02 & 1,8 \\
\hline Madarose total & & 0 & 0 & 0 & 0 & 0 & 0 & 01 & 100,0 & 01 & 0,9 \\
\hline Pterígio inicial & & 07 & 16,3 & 11 & 25,6 & 10 & 23,2 & 15 & 34,9 & 43 & 39,8 \\
\hline Pterígio moderado ou grave & & 01 & 4,5 & 04 & 18,2 & 08 & 36,4 & 09 & 40,9 & 22 & 20,4 \\
\hline Sem comprometimento & & 03 & 16,7 & 04 & 22,2 & 03 & 16,7 & 08 & 44,4 & 18 & 16,7 \\
\hline
\end{tabular}

Observando a distribuição dos comprometimentos objetivos dos olhos e anexos por idade, notamos que a incidência aumenta a partir dos quinze anos e concentra-se até os 55 anos, mesmo para as patologias como diminuição da acuidade visual. Deve destacar-se que a população acometida integra o grupo economicamente ativo do país. Este fato deve ser do conhecimento dos profissionais que atuam no progra- ma, pois o não acompanhamento e tratamento destes quadros, pode significar, em um primeiro momento, causa de absenteísmo ao trabalho e posteriormente, o agravamento pode levar à cegueira que significa elevado ônus para a sociedade, além de causar transtornos a integração social do indivíduo e fortalecer o estigma da hanseníase. (ver Tabela 3). 
Tabela 3 - Distribuição dos comprometimentos dos olhos e anexos por faixa etária - PO-HECU 1991.

\begin{tabular}{|c|c|c|c|c|c|c|c|c|c|c|c|c|c|c|c|c|c|c|}
\hline \multirow{2}{*}{$\begin{array}{l}\text { Faixa Etária } \\
\text { Comprometimentos }\end{array}$} & \multicolumn{2}{|r|}{5 a 10} & \multicolumn{2}{|c|}{10 a 15} & \multicolumn{2}{|c|}{15 a 25} & \multicolumn{2}{|c|}{25 a 35} & \multicolumn{2}{|c|}{35 a 45} & \multicolumn{2}{|c|}{45 a 55} & \multicolumn{2}{|c|}{55 a 65} & \multicolumn{2}{|c|}{$\geqslant 65$} & \multicolumn{2}{|r|}{ Total } \\
\hline & № & $\%$ & № & $\%$ & № & $\%$ & № & $\%$ & № & $\%$ & № & $\%$ & № & $\%$ & № & $\%$ & № & $\%$ \\
\hline \Schirmer & 03 & 6,2 & 03 & 6,2 & 07 & 14,6 & 07 & 14,6 & 05 & 10,4 & 11 & 23,0 & 07 & 14,6 & 05 & 10,4 & 48 & 44,4 \\
\hline Domi & 0 & 0 & 0 & 4, & 02 & 9,1 & 06 & & 6 & & 3 & & 04 & 18,2 & 0 & & 22 & 0.4 \\
\hline & & ( & & 9 & 0 & & 04 & & 5 & 22 & 6 & & 1 & 5 & 04 & & 2 & \\
\hline $\mathbf{N}$ & c & c & ( & 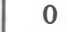 & 03 & 16,7 & 03 & 16 & 1 & 5 , & 4 & & 2 & 1. & 5 & & 8 & 6,7 \\
\hline & 0 & 0 & 01 & 4,2 & 03 & & 01 & 4, & a & & 9 & & 10 & & 24 & & 4 & $2+3$ \\
\hline & $\mathbf{U}$ & 0 & 0 & C & 01 & 6 & 01 & 6,3 & 01 & 6,3 & 06 & & 04 & & 03 & & 6 & 4.8 \\
\hline & 0 & 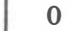 & & & 1 & & & ( & & 0 & 0 & & 01 & 33,4 & 01 & 33,4 & 3 & 2 \\
\hline & 0 & 0 & ( & 0 & 02 & 66,7 & & 0 & & 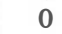 & 01 & & 0 & 0 & 0 & 0 & 3 & 2,8 \\
\hline & 01 & 50,0 & 0 & 0 & 0 & 0 & 0 & 0 & 0 & 0 & 01 & 50 & 0 & 0 & 0 & 0 & 12 & 1,8 \\
\hline & & & & & & & & & & 0,0 & & & & & & & & \\
\hline & 0 & 0 & 02 & 4,6 & 07 & & 08 & & 10 & 23,3 & & & 04 & 9,3 & 02 & 4 , & 43. & 40,0 \\
\hline & 0 & 0 & 0 & 0 & 05 & 22 & 06 & 27, & 02 & 9, & 03 & 13,6 & 03 & 13,6 & 03 & 13,6 & 22 & 26,3 \\
\hline Sem comprometimento & 02 & 22,2 & 07 & 39,0 & 04 & 22,2 & 03 & 16,7 & 01 & 5,5 & 01 & 5,5 & 0 & 0 & 0 & 0 & 18 & 16,7 \\
\hline
\end{tabular}

Observando a distribuição dos comprometimentos oculares pelo tempo de doença percebemos que a incidência é elevada mesmo quando o tempo de doença é curto. A distribuição da incidência tende a discreta homogeinidade, com predominância no período mais curto $\left(\leqslant 1^{\mathrm{a}}\right)$. Este fato parece opor-se aos achados de ROGERS $^{2}$, RAJAN $^{6}$, cuja observação aponta para o aumento da incidência em tempos de doença superior a 5 anos. Alguns fatores podem ser apresentados como justificativa para esta diferenciação: protocolo estabelecido em cada tra- balho; material e método utilizados; população com características bem diferenciadas e por vezes tendenciosa. No entanto, um questionamento surge frente a este dado: a instalação de quadros oculares ocorre desde o início da doença, havendo apenas exacerbação ao longo da evolução ou a instalação ocorre ao longo da evolução da doença ? Este fato, ainda que não bem explicado, deve reforçar a necessidade de acompanhamento precoce e sistemático do aperelho visual (ver Tabela 4).

Tabela 4 - Distribuição dos compromentimentos objetivos dos olhos e anexos por tempo de doença - PO-HECU - 1991.

\begin{tabular}{|c|c|c|c|c|c|c|c|c|c|c|c|}
\hline \multirow[b]{2}{*}{ Comprometimentos } & \multirow[t]{2}{*}{ Tempo } & \multicolumn{2}{|c|}{$\leqslant \quad 1 a$} & \multicolumn{2}{|c|}{$1 a \vdash 2 a$} & \multicolumn{2}{|c|}{$2 a \vdash 3 a$} & \multicolumn{2}{|r|}{$3 a$} & \multicolumn{2}{|c|}{ Total } \\
\hline & & № & $\%$ & № & $\%$ & № & $\%$ & № & $\%$ & № & $\%$ \\
\hline$\downarrow$ Schirmer & & 15 & 32,6 & 16 & 34,8 & 05 & 10,9 & 10 & 21,7 & 46 & 45,1 \\
\hline Paresia & & 07 & 36,8 & 05 & 26,3 & 02 & 10,5 & 05 & 26,3 & 19 & 18,6 \\
\hline I Sensibilidade corneal & & 06 & 30,0 & 07 & 35,0 & 0 & 0 & 07 & 35,0 & 20 & 19,6 \\
\hline Madarose parcial & & 03 & 20,0 & 06 & 40,0 & 02 & 13,3 & 04 & 26,7 & 15 & 14,7 \\
\hline I Ac. Visual p.longe & & 08 & 33,3 & 08 & 33,3 & 02 & 9,4 & 06 & 25,0 & 24 & 23,5 \\
\hline$\downarrow$ Ac. Visual p.perto & & 07 & 43,7 & 07 & 43,7 & 0 & 0 & 02 & 12,6 & 16 & 15,7 \\
\hline Triquiase & & 01 & 33,3 & 01 & 33,3 & 0 & 0 & 01 & 33,3 & 03 & 2,9 \\
\hline Lagoftalmo I (5mm) & & 01 & 100,0 & 0 & 0 & 0 & 0 & 0 & 0 & 01 & 1,0 \\
\hline Cegueira unilateral & & 0 & 0 & 0 & 0 & 01 & 100,0 & 0 & 0 & 01 & 1,0 \\
\hline Madarose total & & 0 & 0 & 01 & 100,0 & 0 & 0 & 0 & 0 & 01 & 1,0 \\
\hline Pterígio inicial & & 20 & 46,5 & 08 & 18,6 & 05 & 11,6 & 10 & 23,3 & 43 & 42,1 \\
\hline Pterígio moderado ou grave & & 06 & 30,0 & 07 & 35,0 & 0 & 0 & 07 & 35,0 & 20 & 19,0 \\
\hline Sem comprometimento & & 06 & 33,3 & 02 & 11,2 & 04 & 22,2 & 06 & 33,3 & 18 & 17,6 \\
\hline
\end{tabular}

\section{CONCLUSÃO}

Ao longo do estudo realizado, percebemos que os comprometimentos oculares hansênicos apresentam elevada prevalência mesmo em grupo de pacientes virgens de tratamento (VT).

Os comprometimentos diagnosticados, foram, em sua maioria, iniciais, de moderada gravidade e reversíveis como diminuição do Schirmer $(44,4 \%)$, paresia $(20,4 \%)$ e diminuição da sensibilidade $(20,4 \%)$. Entretanto, impõe-se que sejam detectados e tratados precocemente, pois a evolução natural dos mesmos, se negligenciados, determinará a cegueira. Autores como SEGHAL, LAMBA ${ }^{6}, \mathrm{CHOYCE}^{8}, \mathrm{LAMBA}^{9}$ situam a hanseníase como causa expressiva de cegueira em países endêmicos, como o Brasil. É interessante analisar a representatividade da cegueira sob dois aspectos fundamentais: para o hanseniano significa verdadeira barreira à integração em seu meio social, especialmente se associada a deformidades físicas em mãos e pés; para a saúde pública representa perpetuação do forte estigma que acompanha a hanseníase, além de interferir no êxito do programa de controle.

Desta forma, urge que o acompanhamento sistemático do aparelho visual torne-se parte integrante do programa de controle da hanseníase. Conforme demonstrado por BRAND ${ }^{3}$, esta ati- 
vidade requer pouco empenho financeiro, pequeno aumento no tempo do atendimento e o treinamento de profissionais de saúde para a atividade, é de curta duração, não sendo exclusividade, neste caso, do especialista.

Assim, propomos como forma de possibili- tar o aumento da demanda, que o enfermeiro, elemento imprescindível da equipe de saúde, assuma o desenvolvimento da atividade pois, além de assumir seu papel integralmente na assistência direta ao paciente, estará abrindo novo : campo de atuação para a categoria.

\section{REFERÊNCIAS BIBLIOGRÁFICAS}

1 COUR TRIGH T, Paul, JOHNSON, Gordon. Prevention of Blindness in leprosy: London. The International Centre eye health, 1988.

2 ROGERS, M. Г. - Lepra prize essay 1982, Lepr. Rev., 55: 60-79, 1984.

3 BRAND, Margareth, JOFFRION, Van. Leprosy of the eye - a general outline, Lepr.Rev. 55: 105-114, 1984.

4 FFY TCHE, Г.J. - Residual sight - threatenig lesions in leprosy patients complecting multidrug therapy and sulphone monotherapy. Lepr. Rev. 62: 35-43, 1991.

5 LIMA, Lauro de Souza Lima. Hospital, Noções de hansenologia. Bauru, 1981.
6 RAJAN, M.A. - Eye in multidrug therapy, Indian Journal of Leprosy. 62(1): 33-38, 1991.

7 SEHGAL, Virendra, LAMBA, Prem. Ocular Changes in leprosy - International Journal of dermato$\operatorname{logy.~29(3):175,~} 1990$.

8 CHOYCE, D.P. - Blindness in leprosy - Trop. Doctor. 3: 15-19, 1973.

9 LAMBA, P.A. Ocular Involvement for leprosy - Indian Journal Ophtalmology. 65: 240-242, 1984.

10 ROLIM, Bárbara, SILVA, Élida Marta - Assistência de enfermagem em prevenção of talmológica nas lesōes oculares hansênicas. Prêmio (2\% lugar) no II ENCENFRIO, 1990.

ASSINE A REBEn E PARTICIPE

ENVIANDO TRABALHOS, RESUMOS DE TESES, RESENHAS DE LIVROS, EXPERIËNCIAS E SUGESTÖES. SUA COLABORAÇĀO E IMPORTANTE! 